\title{
Correction to: Selective galactose culture condition reveals distinct metabolic signatures in pyruvate dehydrogenase and complex I deficient human skin fibroblasts
}

\author{
Damian Hertig $^{1,2,3} \cdot$ Andrea Felser $^{2} \cdot$ Gaëlle Diserens $^{1} \cdot$ Sandra Kurth $^{2} \cdot$ Peter Vermathen $^{1} \cdot$ Jean-Marc Nuoffer $^{2,4} \mathbb{D}$
}

Published online: 19 March 2019

(c) Springer Science+Business Media, LLC, part of Springer Nature 2019

\section{Correction to: Metabolomics (2019) 15:32 \\ https://doi.org/10.1007/s11306-019-1497-2}

The original version of this article contained an error in Table 2. The text in the second header line should read "GAL supernatant" and "GAL Medium" instead of "GLC supernatant" and "GLC Medium". The corrected Table 2 is given below. The original article has been corrected.

Publisher's Note Springer Nature remains neutral with regard to jurisdictional claims in published maps and institutional affiliations.

Damian Hertig and Andrea Felser: shared first author.

Peter Vermathen and Jean-Marc Nuoffer: shared last author.

The original article can be found online at https://doi.org/10.1007/ s11306-019-1497-2.

$\triangle$ Jean-Marc Nuoffer jean-marc.nuoffer@insel.ch

1 Departments of BioMedical Research and Radiology, University of Bern, Bern, Switzerland

2 Institute of Clinical Chemistry, Inselspital, University Hospital Bern, 3010 Bern, Switzerland

3 Graduate School for Cellular and Biomedical Sciences, University of Bern, Bern, Switzerland

4 Department of Paediatrics, Inselspital, University Hospital Bern, Bern, Switzerland 
Table 2 Complete list from integrated buckets of ${ }^{1} \mathrm{H}$ HR-MAS NMR resonances of extracellular metabolites (in arbitrary units) in culture supernatant of Ctrl, PDH, and CI deficient fibroblasts under GLC or GAL culture condition

\begin{tabular}{|c|c|c|c|c|c|c|c|}
\hline GLC supernatant & GLC medium & Ctrl mean & $\pm \mathrm{SD}$ & PDH mean & $\pm \mathrm{SD}$ & CI mean & $\pm \mathrm{SD}$ \\
\hline Nicotinamide & 0.6 & 0.6 & \pm 0.2 & 0.7 & \pm 0.1 & 0.6 & \pm 0.2 \\
\hline Folic acid? Formic acid? & 0.3 & 0.8 & \pm 0.1 & 0.8 & \pm 0.2 & 0.6 & \pm 0.2 \\
\hline Uridine + cytidine & 1.5 & 3.1 & \pm 0.3 & 3.0 & \pm 0.2 & 3.1 & \pm 0.2 \\
\hline Uracil & 1.7 & 1.8 & \pm 0.9 & 1.5 & \pm 0.4 & 1.2 & \pm 0.6 \\
\hline Phenylalanine & 9.6 & 12.6 & \pm 0.6 & 12.5 & \pm 0.6 & 12.8 & \pm 0.5 \\
\hline Tyrosine & 16.6 & 17.3 & \pm 0.6 & 16.8 & \pm 0.9 & 17.4 & \pm 0.6 \\
\hline Glycerophosphocholine & 0.6 & 3.0 & \pm 0.5 & 3.6 & \pm 0.4 & 3.4 & \pm 0.3 \\
\hline Inositol & 10.8 & 12.0 & \pm 0.5 & 11.6 & \pm 0.7 & 12.1 & \pm 0.6 \\
\hline Aspartate & 6.0 & 6.7 & \pm 0.4 & 6.5 & \pm 0.2 & 6.6 & \pm 0.2 \\
\hline Glutamine & 17.4 & 14.4 & \pm 3.5 & 12.8 & \pm 1.6 & 14.2 & \pm 3.8 \\
\hline Glutamate, pyruvic acid & 23.7 & 17.4 & \pm 2.6 & 16.8 & \pm 2.2 & 17.0 & \pm 3.4 \\
\hline Alanine & 17.2 & 20.4 & \pm 1.9 & 19.4 & \pm 1.0 & 19.9 & \pm 1.9 \\
\hline Lactate & 6.3 & 80.1 & \pm 16.7 & 80.2 & \pm 23.2 & 71.3 & \pm 5.9 \\
\hline Isoleucine & 20.6 & 20.3 & \pm 0.8 & 19.8 & \pm 1.3 & 21.0 & \pm 0.8 \\
\hline Valine & 20.6 & 20.3 & \pm 0.8 & 19.7 & \pm 1.1 & 21.0 & \pm 0.9 \\
\hline Leucine & 37.4 & 34.4 & \pm 1.7 & 33.4 & \pm 2.1 & 35.4 & \pm 2.0 \\
\hline Glucose & 31.3 & 25.9 & \pm 2.0 & 24.6 & \pm 3.7 & 26.2 & \pm 2.5 \\
\hline GAL supernatant & GAL medium & Ctrl mean & $\pm \mathrm{SD}$ & PDH mean & $\pm \mathrm{SD}$ & CI mean & $\pm \mathrm{SD}$ \\
\hline Nicotinamide & 0.7 & 0.6 & \pm 0.1 & 0.5 & \pm 0.1 & 0.5 & \pm 0.2 \\
\hline Folic acid? formic acid? & 0.1 & 1.0 & \pm 0.1 & 1.0 & \pm 0.3 & 0.8 & \pm 0.1 \\
\hline Uridine + cytidine & 1.2 & 3.2 & \pm 0.5 & 3.3 & \pm 0.3 & 3.1 & \pm 0.4 \\
\hline Uracil & 0.0 & 2.2 & \pm 1.1 & 2.0 & \pm 0.5 & 1.9 & \pm 0.6 \\
\hline Phenylalanine & 9.7 & 13.0 & \pm 0.8 & 12.6 & \pm 1.0 & 12.8 & \pm 0.9 \\
\hline Tyrosine & 16.9 & 17.7 & \pm 0.8 & 17.0 & \pm 1.2 & 17.3 & \pm 0.9 \\
\hline Glycerophosphocholine & 0.1 & 3.1 & \pm 0.6 & 3.7 & \pm 0.4 & 3.4 & \pm 0.6 \\
\hline Inositol & 13.0 & 13.5 & \pm 0.7 & 12.6 & \pm 0.9 & 13.1 & \pm 1.0 \\
\hline Aspartate & 6.6 & 6.9 & \pm 0.5 & 6.4 & \pm 0.4 & 6.5 & \pm 0.5 \\
\hline Glutamine & 18.3 & 13.8 & \pm 4.0 & 11.8 & \pm 2.0 & 13.7 & \pm 4.0 \\
\hline Glutamate, pyruvic acid & 25.7 & 15.3 & \pm 3.9 & 15.7 & \pm 3.3 & 15.2 & \pm 3.8 \\
\hline Alanine & 18.4 & 19.4 & \pm 2.1 & 18.7 & \pm 1.2 & 19.0 & \pm 1.8 \\
\hline Lactate & 6.9 & 23.5 & \pm 9.0 & 28.4 & \pm 5.7 & 24.6 & \pm 9.7 \\
\hline Isoleucine & 22.0 & 21.1 & \pm 1.1 & 20.1 & \pm 1.8 & 20.9 & \pm 1.2 \\
\hline Valine & 21.7 & 21.1 & \pm 1.1 & 20.2 & \pm 1.7 & 21.1 & \pm 1.2 \\
\hline Leucine & 39.8 & 36.1 & \pm 2.0 & 33.9 & \pm 2.9 & 35.6 & \pm 1.7 \\
\hline Galactose & 50.8 & 52.1 & \pm 2.9 & 49.5 & \pm 4.4 & 50.1 & \pm 3.5 \\
\hline
\end{tabular}

Statistical significance between groups was calculated by two-way ANOVA followed by Benjamini and Hochberg posttest

"?" Metabolite identification needs further confirmation

Ctrl control, $P D H$ pyruvate dehydrogenase, $C I$ complex I

${ }^{*} \mathrm{p}<0.01, \mathrm{Ctrl}$ versus $\mathrm{PDH}$ or $\mathrm{CI}$ in each culture condition

\# $\mathrm{p}<0.01$, GLC versus GAL for Ctrl, PDH or CI 